(C) 1984. The Genetical Society of Great Britain

\title{
THE EFFECT OF LARVAL DENSITY ON AN INVERSION POLYMORPHISM IN THE SEAWEED FLY COELOPA FRIGIDA
}

\author{
R. K. BUTLIN*, P. M. COLLINS AND T. H. DAY \\ Department of Genetics, University of Nottingham
}

Received 20.ix.83

\section{SUMMARY}

\begin{abstract}
The seaweed fly, Coelopa frigida, is polymorphic for two gene arrangements on chromosome I. Inversion frequencies in natural populations are very stable, both geographically and temporally and there is a consistent excess of heterokaryotypes. Laboratory experiments are reported which demonstrate that the egg to adult viability of heterokaryotypes is higher than either homokaryotype. This advantage increases markedly with larval density. Field samples also show a strong correlation between larval density and relative viability, expressed as the excess of heterokaryotypes over Hardy-Weinberg expectations. Larvae of a closely related species, $C$. pilipes, contribute significantly to this density effect in natural populations.
\end{abstract}

\section{INTRODUCTION}

For many years population geneticists have considered heterozygous advantage to be the simplest and best understood mechanism for maintaining genetic polymorphism. In consequence it is surprising that there are only a few convincing examples of loci on which it appears to be operating (Parkin, 1979). The evidence that polymorphisms involving chromosomal inversions are maintained by heterokaryotypic advantage has been consistently more convincing (Lewontin, 1974). However it is an equally consistent finding that the selective forces operating on these polymorphisms are neither simple nor easily understood.

Inversions often involve large numbers of loci and affect many aspects of the phenotype. We might therefore expect a variety of selection pressures to influence inversion frequencies, and heterokaryotypic advantage to be a consequence of any one of these pressures, or of interactions between them. These selective forces are hardly likely to be constants (Kojima, 1971; Clarke, 1979). They might well be influenced by many variables in the physical environment-temperature, humidity, salinity, etc.-and by components of the biotic environment such as predators, parasites or competitors. Competition with other individuals of the same species might also result in fitnesses being influenced by population densities. Such a relationship has been observed in Drosophila pseudoobscura (Dobzhansky, 1947; Birch, 1955), where the fitnesses of chromosome inversion karyotypes differ in crowded and uncrowded conditions. Similarly in $D$. pavani inversion heterokaryotypes have a higher viability than homokaryotypes in crowded, but not uncrowded, laboratory cultures (Budnik et al., 1971). Effects of

\footnotetext{
* Present Address: School of Biological Sciences, University of East Anglia, Norwich.
} 
larval crowding are also seen in experiments involving enzyme polymorphisms (Kojima and Huang, 1972, for esterase-6 in D. melanogaster; Točić and Ayala, 1981, for malate dehydrogenase- 2 in D. pseudoobscura; and Van Delden, 1982, for alcohol dehydrogenase in $D$. melanogaster).

A problem with many of these studies on Drosophila species is the difficulty of relating crowding under laboratory conditions to larval densities in natural populations. As a result, the role of density fluctuations in determining gene (or inversion) frequencies in the field, remains obscure.

The seaweed fly, Coelopa frigida, lives in a clearly defined natural habitat-piles of rotting seaweed on beaches-where it is possible to estimate larval densities (Burnet, 1961). It is polymorphic for a complex rearrangement of chromosome I (Day et al., 1982), with the two alternative sequences designated $\alpha$ and $\beta$. Samples from natural populations show a consistent excess of $\alpha \beta$ heterokaryotypes and deficiency of homokaryotypes (Butlin et al., 1982; Day et al., 1983). Here we report laboratory experiments that demonstrate density dependent viability differences between karyotypes, as well as evidence from field samples for a relationship between larval density and an excess of heterokaryotypes.

\section{Materials AND MEthods}

Karyotypes have been inferred not from chromosomal squashes, but from determination of alcohol dehydrogenase $(A d h)$ genotypes. The $A d h$ locus has been shown to be strongly associated with the $\alpha / \beta$ inversion system by Day et al., (1982). The $A d h$-B allele is always associated with the $\alpha$ sequence and the $A d h-\mathrm{D}$ allele with the $\beta$ sequence. In natural British populations a third allele, $A d h-\mathrm{C}$, is present at a frequency of $0 \cdot 05-0 \cdot 15$. This allele can be associated with either sequence. For this reason we have avoided using animals carrying the $C$ allele in our laboratory experiments, and in samples from natural populations, karyotype frequencies have been inferred from individuals carrying only the $B$ or $D$ alleles.

Inferring karyotypes from $A d h$ genotypes has the advantage of allowing large samples to be scored at any stage of the life history. We consider this advantage outweighs the error introduced by omitting the $A d h-C$ allele (see Day et al., 1982). Methods for electrophoresis have been described by Butlin et al., (1982).

\section{(i) Laboratory experiments}

A sample of larvae, together with the seaweed in which they were living, was collected from Rustington, Sussex (Ordnance Survey grid reference, TV 049014). The larvae were allowed to pupate in the laboratory and the adults aspirated soon after eclosion. Pairs of virgin flies were set up at random in vials containing standard culture medium (Day and Buckley, 1980) and maintained at $27^{\circ} \mathrm{C}$ until an egg batch was laid. Adults were then removed and their $A d h$ genotypes determined.

Only those egg batches laid by parents who were both $A d h-B D$ (and therefore $\alpha / \beta$ heterokaryotypes) were used to establish the replicates in the density experiment. One, five or ten egg batches were added to canisters $(9 \times 9 \times 13 \mathrm{~cm}$ deep) containing $200 \mathrm{~g}$ of fresh medium. These were designated respectively, low, medium and high density cultures. The number of 
eggs used in each culture was counted. In Coelopa frigida hatch rates are uniformly high (about 98-99 per cent-Thompson, 1951; Collins, 1978), and so a reliable estimate was obtained of the initial number of larvae per canister.

Canisters were maintained at $27^{\circ} \mathrm{C}$ in constant darkness. All adults emerging were collected daily, counted and stored at $-20^{\circ} \mathrm{C}$ for subsequent electrophoresis. For the low density replicates the genotypes of all adults were determined. For the medium and high density replicates all daily collections were pooled, and a sample of 72 flies per canister was used for electrophoresis.

\section{(ii) Field samples}

An attempt was made to relate larval densities to the genotype frequencies in a series of seaweed beds. Samples were collected from Whitburn, Tyne and Wear (NZ 408614) on two separate occasions, and from St Mary's Island, Tyne and Wear (NZ 350753) on four occasions during 1981. These two sites are separated by about $15 \mathrm{~km}$ of coastline.

Genotype frequencies were obtained from mass samples collected and analysed by the "adult" method described by Butlin et al., (1982). These data form part of a larger set of samples described elsewhere (Butlin, 1983; Day, Cullen and Butlin, in prep.). Here we have used the ratio of the observed number of $A d h-B D$ individuals to the Hardy-Weinberg expectation as a measure of the heterokaryotypic excess, which is independent of variation in $\alpha$ and $\beta$ frequencies between samples.

Larval densities in the seaweed beds were estimated by a method similar to that of Burnet (1961). A soil corer of internal diameter $4.75 \mathrm{~cm}$ was forced through the bed and into the sand or shingle below. The core of seaweed produced, with the larvae it contained, was placed in a container. Ten cores were taken from random positions in each seaweed bed sampled. The tough nature of large seaweeds made it virtually impossible to standardise the length of the cores. However, the measured depths of the beds were similar.

On return to the laboratory the cores were placed in canisters supplemented with culture medium to reduce larval density and mortality. All flies emerging were collected and counted. In addition to Coelopa frigida, adults of the closely related species Coelopa pilipes were common in some beds.

\section{Results}

\section{(i) Larval densities, survival and genotype frequencies} under laboratory conditions

It is no surprise that larval survival declined markedly with density (table 1). Over 90 per cent of the variation in survival (expressed as proportions of eggs, surviving to adulthood transformed to angles), was accounted for by a linear regression on density (expressed as the number of eggs per canister on a logarithmic scale), $(b=-0.26 \pm 0.02)$. This relationship implies that survival declines exponentially with density. For this reason a logarithmic density scale has been used in all subsequent analyses.

In no replicates did the genotype frequencies among the survivors differ significantly between males and females $\left(\chi_{2}^{2} \leqq 3 \cdot 92, p>0 \cdot 10\right)$. Pooling the 


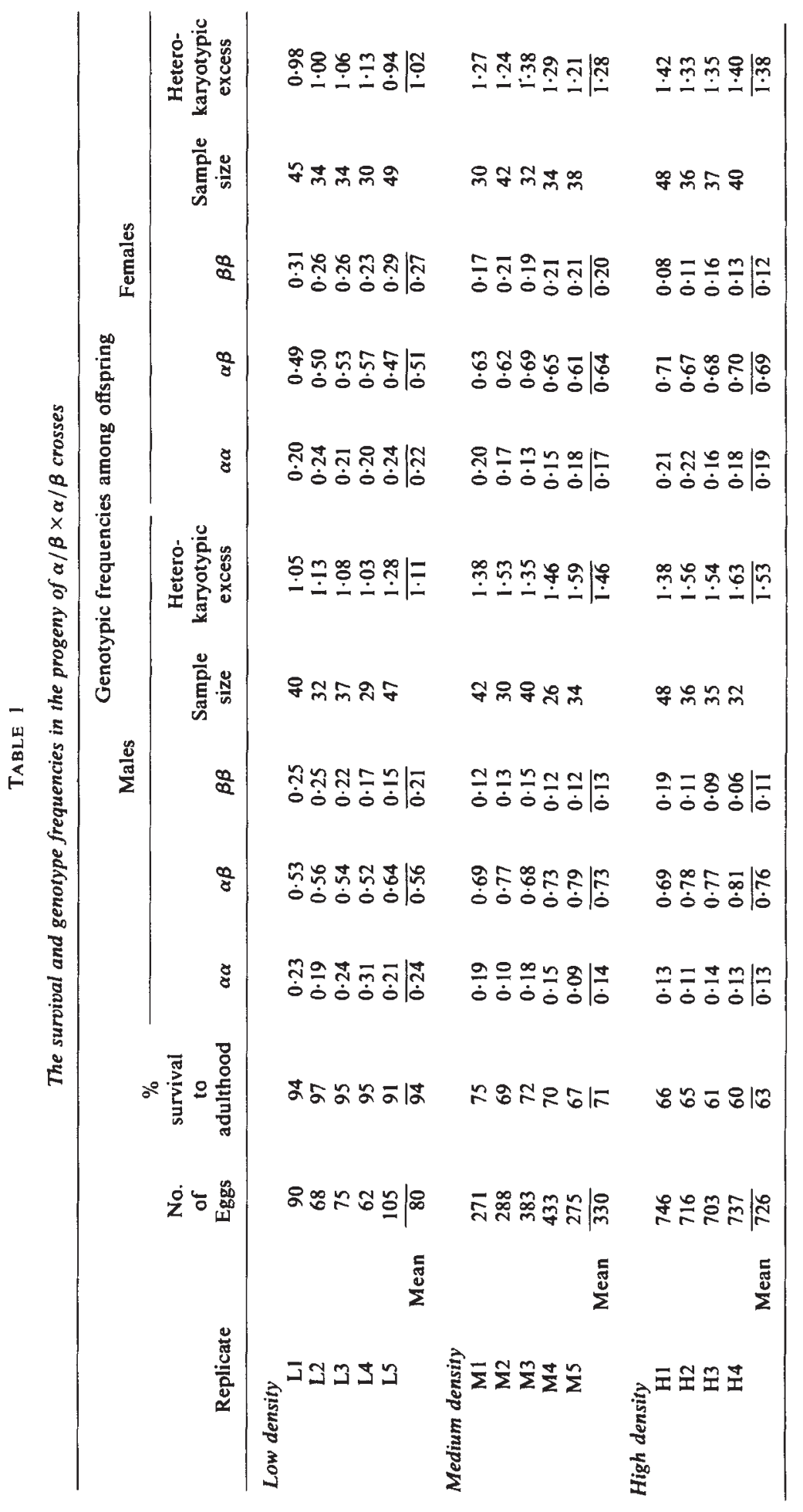


male and female data it can be seen (table 1) that none of the low density replicates differed significantly from the $1: 2: 1$ expectation $\left(\chi_{2}^{2} \leqq 1.06, p>\right.$ $0 \cdot 20$ ). However, all of the medium density replicates departed from Mendelian expectations at the 5 per cent level $\left(\chi_{2}^{2} \geqq 8 \cdot 10\right)$ and all of the high density replicates at the $0 \cdot 1$ per cent level $\left(\chi_{2}^{2} \geqq 14 \cdot 33\right)$. In every case these departures were due to an excess of heterokaryotypes and a deficiency of both homokaryotypes. The proportion of heterokaryotypes varied from $50 \cdot 6$ per cent (in replicate $\mathrm{L}$ 1) to 75.0 per cent (in replicate $\mathrm{H} 4$ ).

There appears to be a strong effect of larval density on the relative viabilities of the karyotypes. This effect was more marked in males than in females, with the males having a consistently higher proportion of heterokaryotypes-particularly at high densities. Regressions of the proportions of heterokaryotypes (in angles) on the logarithm of density explain a large proportion of the variance in both males $(b=0.13 \pm 0.022$, accounting for 74 per cent of variance) and females $(b=0.099 \pm 0.013$, accounting for 84 per cent of variance). However, the slopes of these regressions do not differ significantly $\left(t_{13}=1 \cdot 22, p>0 \cdot 20\right)$.

In males the proportions of the two homokaryotypes showed similar decreases with density (for $\alpha \alpha: b=-0.052 \pm 0.012$; for $\beta \beta: b=-0.045 \pm$ 0.012 ). However in females the reduction in viability was more marked for $\beta \beta$ 's $(b=-0.064 \pm 0.010)$ than for $\alpha \alpha$ 's $(b=0.017 \pm 0.009)$. These slopes differ significantly at the 1 per cent level $\left(t_{26}=3 \cdot 49\right)$.

The relative viabilities of the genotypes are summarised in table 2 . It would appear that, at least under laboratory conditions, the homokaryotypes are markedly less fit than the heterokaryotypes, and that at high densities the selective mortality is very great indeed.

TABLE 2

Relative viabilities of genotypes in laboratory culture

\begin{tabular}{|c|c|c|c|c|c|c|}
\hline \multirow[b]{2}{*}{ Density } & \multicolumn{3}{|c|}{ Males } & \multicolumn{3}{|c|}{ Females } \\
\hline & $\alpha \alpha$ & $\alpha \beta$ & $\beta \beta$ & $\alpha \alpha$ & $\alpha \beta$ & $\beta \beta$ \\
\hline Low & 0.83 & 1 & 0.73 & 0.87 & 1 & $1 \cdot 09$ \\
\hline Medium & 0.40 & 1 & 0.35 & 0.52 & 1 & 0.63 \\
\hline High & 0.33 & 1 & 0.32 & 0.56 & 1 & 0.34 \\
\hline
\end{tabular}

(ii) The densities and distribution of larvae "in the field"

In order to enquire whether the differential mortality observed under laboratory conditions also occurs in natural populations, we need to estimate larval densities "in the field". This is not easily done. Coelopa lives in vast, heterogeneous piles of marine algae cast up on the shore. A "patch" of woody stipe of Laminaria hyperborea is physically very different from a "patch" of Fucus serratus frond. A further complication to sampling is that Coelopa frigida lays its eggs in batches in hot spots within the seaweed bed. This results in a high degree of aggregation of larvae (Burnet, 1961).

We have used the technique of "random coring" to provide an approximate estimate of larval density. Vertical cylindrical cores of seaweed were taken at random from ten widely spaced sites in the bed. The larvae were 
grown to adulthood in the laboratory, and were then counted and classified as Coelopa frigida or $C$. pilipes. This technique yields an estimate of average density, as well as a measure of aggregation of both $C$. frigida and $C$. pilipes.

The results, expressed as the number of animals observed in a unit weight of seaweed, are compared with the number of adults surviving in laboratory cultures (see table 3 ). It can be seen that the densities of larvae

TABLE 3

Comparison of Coelopa densities in laboratory experiments and in natural populations. WH refers to samples from Whitburn and SM to samples from St Mary's Island

\begin{tabular}{|c|c|c|c|c|c|}
\hline \multirow{2}{*}{$\begin{array}{l}\text { Sample } \\
\text { laboratory } \\
\text { cultures }\end{array}$} & \multicolumn{2}{|c|}{$\begin{array}{l}\text { Mean density (Range) } \\
\text { (larvae/gm) }\end{array}$} & \multicolumn{2}{|c|}{ Aggregation } & \multirow{2}{*}{$\begin{array}{l}\text { Hetero- } \\
\text { karyotypic } \\
\text { excess in } \\
\text { C. frigida }\end{array}$} \\
\hline & C. frigida & C. pilipes & C. frigida & C. pilipes & \\
\hline Low & $0.4(0.29-0.48)$ & Not present & - & - & $1 \cdot 02$ \\
\hline Medium & $1.2(0.92-1.52)$ & Not present & - & - & $1 \cdot 28$ \\
\hline $\begin{array}{l}\text { High } \\
\text { Natural } \\
\text { populations }\end{array}$ & $2 \cdot 3(2 \cdot 14-2 \cdot 46)$ & Not present & - & - & $1 \cdot 38$ \\
\hline WH $(18 / 3)$ & $0 \cdot 1(0-0.63)$ & $0 \cdot 1(0-0 \cdot 50)$ & $6 \cdot 0$ & 1.7 & $1 \cdot 12$ \\
\hline $\operatorname{SM}(18 / 3)$ & $0.6(0.30-2.43)$ & $0.004(0-0.03)$ & $18 \cdot 1$ & $2 \cdot 1$ & $1 \cdot 12$ \\
\hline WH $(14 / 5)$ & $0 \cdot 1(0-0.50)$ & $0.5(0-1.23)$ & 6.6 & $1 \cdot 2$ & $1 \cdot 15$ \\
\hline SM $(14 / 5)$ & $2 \cdot 2(0.03-14.40)$ & $0.008(0-0.07)$ & $174 \cdot 2$ & 1.8 & 1.25 \\
\hline SM $(27 / 8)$ & $1 \cdot 6(0-5 \cdot 53)$ & $0 \cdot 3(0-2 \cdot 30)$ & $53 \cdot 0$ & $1 \cdot 7$ & $1 \cdot 27$ \\
\hline $\operatorname{SM}(4 / 11)$ & $1.0(0 \cdot 23-3 \cdot 80)$ & $0.006(0-0.07)$ & 27.9 & $2 \cdot 3$ & $1 \cdot 18$ \\
\hline
\end{tabular}

under natural conditions are very variable, but are nevertheless broadly comparable to the densities used in the laboratory experiments. We conclude then that animals in natural populations are experiencing densities at least as high as those shown to be associated with selective mortality of homokaryotypes under laboratory conditions.

In passing, we should also comment on the distribution of larvae in seaweed beds. If animals are distributed at random then the numbers of larvae per random core should follow a Poisson distribution, and the variance should equal the mean in any set of cores. The ratio of variance to the mean $\left(s^{3} / \bar{x}\right)$ can therefore be used to investigate the aggregation or dispersal of larvae. In all core series the ratio was much greater than 1.0 for $C$. frigida (table 3), indicating that larvae of this species have a highly clumped distribution. For $C$. pilipes the ratios are much nearer to $1 \cdot 0$. These observations are in full agreement with those of Burnet (1961). It seems likely that the difference in distributions is a consequence of the different patterns of oviposition. $C$. frigida females lay their eggs in clutches of 70-80 eggs, whereas $C$. pilipes females lay their eggs singly, in a similar fashion to Drosophila melanogaster females.

\section{(iii) Larval densities and genotype frequencies} in natural populations

We have presented evidence that under laboratory conditions there is an association between larval density and the mortality of homokaryotypes. 
If such an association exists in natural seaweed beds, then we would expect populations growing at high densities to exhibit an excess of heterokaryotypes. From each of the six populations in which larval densities were studied, a large sample was taken to estimate genotype frequencies. Heterokaryotypic excess is expressed as the ratio of observed numbers to those expected on the basis of Hardy-Weinberg expectations. The ratios are given in table 3 .

Before analysis both variables have been transformed to a logarithmic scale. This was necessitated by the clear relationship between the mean and variance of larval densities, and by the skewness of the ratio used to measure heterokaryotypic excess. A regression of heterokaryotypic excess on density of $C$. frigida explains 45 per cent of the variance, but the slope is not significantly different from zero $(b=0.0168 \pm 0.0074, p>0.05)$. However, with the density of $C$. pilipes as a second independent variable, 84 per cent of the variation is explained and this is a significant improvement ( F1, $3=10.9, p<0.05)$. With this model both regression coefficients are significantly different from zero (for $C$. frigida $b=0.024 \pm 0.0046, p<0.05$; for $C$. pilipes $b=0.015 \pm 0.0047, p<0.05)$.

This result is in full agreement with the prediction from the laboratory experiments that heterokaryotypic excess would be greatest at high larval densities. It suggests that viability differences between the karyotypes are responsible, in part at least, for the observed departures from HardyWeinberg expectations in natural populations (Butlin et al., 1982), and that these differences are accentuated under conditions of intense larval competition. In addition, the significant contribution of $C$. pilipes larvae to the density effect in $C$. frigida, indicates that the relative selective values of the karyotypes are influenced by inter- as well as intraspecific competition.

\section{Discussion}

Our laboratory experiments showed a marked drop in larval survival with increasing density. High densities of Coelopa larvae also produce small adult flies with long egg to adult development times (Collins, 1978, Butlin and Day, in preparation). These relationships are typical of competition of the "scramble" type which is common in Dipteran larvae (for example: Lucilia, Nicholson, 1957; Drosophila, Bakker, 1961, Hughes, 1980; Musca, Haupt and Busvine, 1968).

The increasing density, and thus competition, was accompanied by a very marked change in the relative viabilities of the chromosome I karyotypes. $\alpha \beta$ larvae had the highest viability at all densities but their advantage increased as density increased. At the highest densities the viabilities of both homokaryotypes were reduced to less than half that of the heterokaryotypes. This suggests that $\alpha \beta$ individuals are in some way better competitors. Such an advantage could arise in many ways. For example the heterokaryotypes may be more efficient at feeding, more efficient in conversion of food to body weight, more tolerant of toxic residues in the medium, or simply able to pupate successfully at a lower body weight. The fact that the effect of density is more marked in males than in females, especially for the $\alpha \alpha$ karyotype, may provide a clue to the important factor. Differences in both development rate and adult size are associated with the chromosome I karyotype in males, but not in females (Day et al., 1980; Butlin et al., 1982). 
In both cases $\alpha \beta$ individuals are intermediate and thus may represent some optimum combination of growth rate and pupation time. The relationships between density, development time and size have been investigated further by Butlin (1983) who has also carried out additional experiments on viability. All of these experiments showed a higher viability for the heterokaryotype than either homokaryotype (Butlin and Day-in preparation).

Heterokaryotypic advantage in viability under competitive conditions of the strength observed in these laboratory experiments, has the potential to explain both the uniformity of inversion frequencies and the consistent heterokaryotype excess observed in natural populations (Butlin et al., 1982; Day et al., 1983). However there are other possible explanations for these observations. It would be difficult to. demonstrate directly that there were viability differences between karyotypes under natural conditions. However, the laboratory results make a clear prediction that relative viabilities are dependent on larval density and this prediction has been tested. The field samples show a strong relationship between larval density and heterokaryotype excess relative to Hardy-Weinberg expectations. This indicates that viability differences between karyotypes may be at least a major selective agent producing the observed departure from expectation and are, consequently, likely to be important in maintaining the inversion polymorphism.

An additional feature of the field results was the contribution of both Coelopa frigida and $C$. pilipes larvae to the effect of density on relative viabilities. Both contributions were significant but the effect of $C$. pilipes was less than that of $C$. frigida. These results suggest that the two closely related species compete for some limiting resources in the seaweed beds. Furthermore, they suggest that heterokaryotypes in $C$. frigida are not only better intraspecific competitors, but also better interspecific competitors than homokaryotypes. It is likely that the effect of $C$. pilipes larvae may be less than that of $C$. frigida larvae because resource requirements overlap more within species than between species. These observations on natural populations need to be interpreted with care because of the small number of samples studied. However, they do provide an important confirmation of the relevance of the laboratory results and suggest an interesting area of investigation into interspecific interactions.

Density dependent selection may be a common feature in populations whose numbers are regulated by density dependent factors (Clarke, 1972), and may play a part in the maintenance of widespread genetic polymorphism. Recently, interest in density dependence has been stimulated by work on the related phenomenon of frequency dependent selection (Clarke, 1979). As a result there have been many studies of the effect of density on selective values in the laboratory (Kojima and Huang, 1972; Točić and Ayala, 1981; Van Delden, 1982; De Benedictus, 1977; Nassar et al., 1973, Nassar, 1979). This work, mainly with Drosophila, suffers from the disadvantage that densities cannot easily be measured in the field. The data presented here demonstrate that in Coelopa frigida selective values vary with larval competition not only under controlled laboratory conditions but also in natural populations.

Acknowledgments. We are grateful to Sally Cullen for help in collecting samples and to Sue Miles for technical assistance. R. K. Butlin was supported by an SRC Studentship. 


\section{RefERENCES}

BAKKER, K. 1961. An analysis of factors which determine success in competition for food amongst larvae of Drosophila melanogaster. Arch. Neer. Zool., 14, 200-281.

BIRCH, L. C. 1955. Selection in drosophila pseudoobscura in relation to crowding. Evolution, 9, 389-399.

BUDNIK, M., BRNCIC, D. AND KOREF-SANTIBANEZ, S. 1971. The effects of crowding on chromosomal polymorphism of Drosophila pavani. Evolution, 25, 410-419.

BURNET, B. 1961. On the distribution of recessive embryonic lethals in a natural population of Coelopa frigida (Fab.). Genet. Res. Camb., 2, 249-271.

BUTLIN, R. K., COLLINS, P. M., SKEVINGTON, S. J. AND DAY, T. H. 1982. Genetic variation at the alcohol dehydrogenase locus in natural populations of the seaweed fly, Coelopa frigida. Heredity, 48, 45-55.

BUTLIN, R. K. 1983. The maintenance of an inversion polymorphism in Coelopa frigida. Ph.D Thesis, University of Nottingham.

ClARKe, B. 1972. Density-dependent selection. Amer. Nat., 106, 1-13.

ClARKe, B. 1979. The evolution of genetic diversity. Proc. R. Soc. Lond. B., 205, 453-474.

COLLINS, P. M. 1978. Studies on genetic polymorphism in Coelopa frigida. Ph.D. Thesis, University of Nottingham.

DAY, T. H. AND BUCKLEY, P. A. 1980. Alcohol dehydrogenase polymorphism in the seaweed fly, Coelopa frigida. Biochem. Genet., 18, 727-742.

DAY, T. H., DOBSON, T., HILlIER, P. C., PARKIN, D. T. AND ClARKE, B. 1980. Different rates of development associated with the alcohol dehydrogenase locus in the seaweed fly, Coelopa frigida. Heredity, 44, 321-326.

DAY, T. H., DOBSON, T., HILLIER, P. C., PARKIN, D. T. AND CLARKE, B. 1982. Associations of enzymic and chromosomal polymorphisms in the seaweed fly, Coelopa frigida. Heredity, $48,35-44$.

DAY, T. H., DAWE, C., DOBSON, T. AND HILlIER, P. C. 1983. A chromosomal inversion polymorphism in Scandinavian populations of the seaweed fly, Coelopa frigida. Hereditas. In Press.

DE BENEDICTIS. P. A. 1977. Studies on the dynamics of genetically variable populations. I Frequency and density-dependent selection in experimental populations of Drosophila melanogaster. Genetics, 87, 343-356.

DOBZHANSKY, T. 1947. Genetics of natural populations. XIV A response of certain gene arrangements in the third chromosome of Drosophila pseudoobscura to natural selection. Genetics, 32, 142-160.

HAUPT, A. AND BUSVINE, J. R. 1968. The effect of overcrowding on the size of houseflies (Musca domestica L.). Trans. R. ent. Soc. Lond., 120, 297-311.

HUGHES, G. 1980. Larval competition in serially transferred populations of Drosophila melanogaster. Oecologia, 45, 396-403.

KOJIMA, K. 1971. Is there a constant fitness value for a given genotype? No! Evolution, 25 , 281-285.

KOJIMA, K. AND HUANG, S. L. 1972. Effects of population density on the frequency-dependent selection in the Esterase-6 locus of Drosophila melanogaster. Evolution, 26, 313-321.

LEWONTIN, R. C. 1974. The Genetic Basis of Evolutionary Change. Columbia University Press, New York.

NASSAR, R. 1979. Frequency-dependent selection at the lap locus in Drosophila melanogaster. Genetics, 91, 327-338.

NASSAR, R., MUHS, H. J. AND COOK, R. D. 1973. Frequency-dependent selection at the Payne inversion in Drosophila melanogaster. Evolution, 27, 558-564.

NICHOLSON, A. J. 1957. The self adjustment of populations to change. Cold Spring Harb. Symp. Quant. Biol., 22, 153-173.

PARKIN, D. T. 1979. An Introduction to Evolutionary Genetics. Edward Arnold, London.

THOMPSON, U. 1951. Studies on the genetics and ecology of Coelopa frigida (Fab.). Ph.D. Thesis, University of Durham.

TOČIĆ, M. AND AYALA, F. J. 1981. Density-and frequency-dependent selection at the $M d h-2$ locus in Drosophila melanogaster. Genetics, 97, 679-701.

VAN DELDEN, w. 1982. The alcohol dehydrogenase polymorphism in Drosophila melanogasterselection at an enzyme locus. Evol. Biol., 15, 187-222. 